\title{
Clinical Significance of MicroRNA-155 Regulated Autophagy and Apoptosis by targeting gene Rictor/Fos in Gastric Cancer Progression
}

\section{Gang Chen}

the second hospital of Lanzhou University

Yu Li

the second hospital of Lanzhou University

\section{Zhijian Ren}

the second hospital of Lanzhou University

\section{Yanmei Gu}

Key laboratory of digestive system tumors of Gansu Province

\section{Futian Tang}

Lanzhou University Second Hospital

\section{Jie Mao}

the second hospital of Lanzhou University

Junmin Zhu

the second hospital of Lanzhou University

\section{Ling Wang}

key laboratory of digestive system tumors of Gansu Province

Yumin Li ( $\square$ liym@lzu.edu.cn )

Lanzhou University https://orcid.org/0000-0002-9267-1412

\section{Primary research}

Keywords: microRNA-155, gastric cancer, autophagy, apoptosis, Rictor, Fos

Posted Date: May 12th, 2020

DOI: https://doi.org/10.21203/rs.3.rs-18875/v2

License: (c) (i) This work is licensed under a Creative Commons Attribution 4.0 International License. Read Full License

Version of Record: A version of this preprint was published at Nanoscience and Nanotechnology Letters on April 1st, 2020. See the published version at https://doi.org/10.1166/nnl.2020.3144. 


\section{Abstract}

BACKGROUND Microrna-155 plays an important role in the pathogenesis, progression and treatment of various cancers. It is abnormally expressed in gastric cancer, but its expression level, mechanism and significance are controversial in different studies. So we make the study to explore the expression level, significance and mechanism of microRNA-155 on gastric cancer.

METHODS Target genes of microRNA-155 in TargetScan and mirDB databases were analyzed by Wayne Mapping, Enrichr database, String database, TIMER database. Forty-three pairs of cancer tissues and adjacent tissues were collected to extract total RNA and protein. Expression of MicroRNA-155, Rictor, Fos, Beclin1, LC3, caspase3 and caspase9 were measured by qRT-PCR and western blot. The relationship between gene expression and clinicopathological factors were analyzed. SPSS 23.0 was used for statistical analysis.

RESULTS A total of 700 (miRDB) and 556 (TargetScan) target genes were obtained and 280 genes were in the intersection of Wayne Mapping, 49 of them had a target score of 90 or more. GO and KEGG analysis revealed that they were related to autophagy or apoptosis pathway. Rictor and Fos were selected as research objects. Thirty-two cases showed high microRNA-155 expression (group H) and 11 cases showed low expression (group L). Twelve patients had high Rictor expression and 31 patients had low expression; Thirteen cases had roughly normal Fos expression and 30 cases had low or negative expression; Thirtythree cases had high Beclin1/LC3 expression and 10 cases had low expression; Ten cases had high caspase3/caspase9 expression and 33 cases had low expression. According to the results of immunohistochemistry and western blot, Rictor, Fos, caspase3 and caspase9 were low expressed while Beclin1 and LC3 were high expressed in group H. However, all the six genes had no significant difference in group L.

CONCLUSIONS The abnormal expression of microRNA-155 may indicate the occurrence of gastric cancer and its expression level is negatively correlated with clinical stage of cancer. The down-regulated expression of Rictor and Fos, enhancement of autophagy and weakening of apoptosis may be related to the overexpression of microRNA-155. MicroRNA-155 may promote the progression of gastric cancer by promoting autophagy and inhibiting apoptosis.

\section{Background}

MicroRNAs, a class of single-stranded non-coding RNA, are widespread in various organisms, regulate gene expression at post-transcriptional level ${ }^{[1-4]}$, and also play important roles in inflammation, tumorigenesis and progression ${ }^{[5-7]}$. MicroRNA-155 is located on chromosome 21 q21 and aberrantly expressed in a variety of solid tumors. MicroRNA-155 is mainly involved in pathological processes such as inflammation, immunity, tumorigenesis and progression, and plays an important role in the regulation of a variety of key activities including autophagy and apoptosis ${ }^{[8,9]}$.

Autophagy and apoptosis are important life activities in organisms, which play complex and important roles in the development and progression of cancer. Autophagy was discovered by Ashford and Porter in 1962, 
and Yoshinori Ohsumi further elucidated the mechanism ${ }^{[10]}$. Autophagy is an important pathway for cells to remove abnormal proteins, senesce or damage organelles under stress. Meanwhile, insufficient of autophagy will cause carcinogenesis by accumulating several carcinogenic factors ${ }^{[11,12]}$; Hypoxia or starvation can induce autophagy to scavenge the free radicals in cells for avoiding cell damage or to recycle nutrients to maintain cell survival ${ }^{[13-15]}$; Furthermore, autophagy has also been found to cause chemotherapy resistance ${ }^{[16-20]}$. Apoptosis, an actively occurring programmed cell death under the control of apoptotic genes, was first proposed in 1972 by Kerr et al ${ }^{[21]}$. Apoptosis is a fundamental phenomenon of life and is widely involved in life processes such as embryonic development, removal of senescent and diseased cells ${ }^{[22]}$.

A lot of studies have been carried out to explore autophagy, apoptosis and the incidence, progression, metastasis, recurrence and chemotherapy resistance of gastric cancer. Some studies have shown that microRNA-155 is abnormally expressed in gastric cancer and widely involved in the pathological process of gastric cancer via modulation the cell proliferation, apoptosis, invasion, metastasis and other processes ${ }^{\text {[23- }}$ 26]. Additionally, microRNA-155 modulate autophagy levels through adjusting the expression of Rheb, which plays an important role in autophagy initiation ${ }^{[27,28]}$. However, the effect of autophagy for tumor and the mechanisms are not clear enough or even controversial. Herein, we reported this study to further explore the expression level and influencing factors of microRNA-155 in gastric cancer. We also further studied the mechanism of microRNA-155 in the regulation of autophagy and apoptosis as well as the mechanism in the progression of gastric cancer.

\section{Methods}

\section{microRNA-155 target genes prediction and signal pathway analyses}

We utilized TargetScan database and mirDB database to select validated target genes of microRNA-155. The target genes both contained by the two databases were filtrated by Venn Mapping, then they were ranked by target score of mirDB, we choose the genes that scored 90 or more as the range of selected target genes. We take the genes contained in the crossover set to make GO (Gene ontology, GO) analysis and KEGG (Kyote Encyclopedia of Gene and Genome, KEGG) analysis in Enrichr database. One autophagy related gene and one apoptosis related gene were selected based on related literature, furthermore, the two selected genes should show significant differences in expression level between tumor tissues and normal tissues. Then we construct the protein interaction network for the two selected genes in String database.

\section{Clinical samples}

Forty-three gastric cancer tissues and adjacent tissues were obtained during surgery. All patients underwent radical gastrectomy at The Second Hospital of Lanzhou University from July 1st 2017 to June 31st 2019. Samples were placed in liquid nitrogen within 5 minutes of release and then quickly transferred to $-80^{\circ} \mathrm{C}$ refrigerator. Based on the clinicopathologic criteria, all patients were diagnosed as gastric cancer. Total data of patient including age, gender, histological grade, lymph node metastasis, depth of tumor invasion, nerve/venous invasion, Lauren type, Borrmann type and clinical stage were obtained from clinical and 
pathologic records. None of them received neoadjuvant chemotherapy or radiotherapy before surgery. Written informed consent was obtained from each patient and the study protocol was conformed to ethical guidelines of the 1975 Declaration of Helsinki as reflected in a priori approval by the ethics committee of the Second Hospital of Lanzhou University.

\section{Total RNA and protein isolation and quality analysis}

Total RNA of frozen tissues were extracted using Trizol (Invitrogen, American) method according to the manufacturer's instructions. Concentrations and purity of the RNA samples were assayed by electrophoresis and spectrophotometric methods. Total protein were extracted using Protein kit (Beyotime, China) according to the manufacturer's instructions. Concentrations and purity of samples were assayed using bicinchonininc acid (Solarbio, China) method.

\section{MicroRNA-155 and mRNAs quantification by qRT-PCR}

MiRNA/mRNA quantification were tested by qRT-PCR (quantitative real time polymerase chain reaction, qRTPCR). SYBR green qRT-PCR assay was used for miRNA/mRNA quantification. Total RNA containing was reversely transcripted to cDNA using miScript Reverse Transcription kit (Takara, Japan) according to the manufacturer's instructions. QRT-PCR was performed with PCR kit (Takara, Japan) in ABI 7500 Real-time PCR system. Each reaction was performed in a final volume of $20 \mu \mathrm{l}$ containing $1 \mu \mathrm{l}$ of $\mathrm{CDNA}, 0.4 \mu \mathrm{l}$ of each primer and 10 $\mathrm{ll} 1 \mathrm{X}$ SYBR Green PCR Master mix. The amplification program for microRNA-155 was: denaturation at $95^{\circ} \mathrm{C}$ for $15 \mathrm{~min}$, followed by 40 cycles of $95^{\circ} \mathrm{C}$ for $34 \mathrm{sec}, 60^{\circ} \mathrm{C}$ for $20 \mathrm{sec}$. The amplification program for mRNAs was: denaturation at $95^{\circ} \mathrm{C}$ for $30 \mathrm{sec}, 95^{\circ} \mathrm{C}$ for $5 \mathrm{sec}, 60^{\circ} \mathrm{C}$ for $34 \mathrm{sec}$. Each sample was run in triplicates for analysis. The expression levels were normalized to RNU6B or GAPDH. Relative gene expression was calculated as $2^{-(C T m i R N A-C T R N U 6 B ~ R N A)}$. Six patients were randomly selected with random number table from the miR-microRNA-155 high-expression group (group H) and the low-expression group (group L) for Immunohistochemical staining and Western blot analysis.

\section{Immunohistochemical staining}

Expression of genes studied were detected using the UltraSensitive ${ }^{\mathrm{TM}} \mathrm{SP}$ kit-9701 (Fuzhou, China), and the reaction product was visualized with diaminobenzidine. Following deparaffinization in xylene and rehydration, tissue array blocks were incubated in $3 \% \mathrm{H}_{2} \mathrm{O}_{2}$ for 12 min to block endogenous peroxidase activity and then washed three times with phosphate-buffered saline. The blocks were incubated in normal goat serum (Fuzhou, China) for $15 \mathrm{~min}$ at room temperature, and the Corresponding antibody were applied at $4^{\circ} \mathrm{C}$ overnight. Following a 3-min incubation with $\mathrm{DAB}$, the blocks were counterstained with hematoxylin and rinsed with tap water. After that, blocks were immediately dehydrated by sequential immersion in gradient ethanol and xylene and mounted with Permount onto cover slips. Images were obtained under a light microscope (IX53+DP74, Olympus, Japan) .

\section{Western blot}


Protein samples were separated by $12 \%$ SDS-PAGE and transferred to PVDF membranes. After blocked $2 \mathrm{~h}$ with 5\% milk, the PVDF membranes were incubated with Rictor (mouse anti-human monoclonal antibody; 1:1,000, Santa Cruz), Fos (mouse anti-human monoclonal antibody; 1:1,000, Santa Cruz), Beclin1 (mouse anti-human monoclonal antibody; 1:1,000, Santa Cruz), LC3 (mouse anti-human monoclonal antibody; 1:1,000, Santa Cruz), caspase-3 (mouse anti-human monoclonal antibody; $1: 1,000$, Santa Cruz) and caspase-9 (mouse anti-human monoclonal antibody; 1:1,000, Santa Cruz) overnight at $4^{\circ} \mathrm{C}$. Following extensive washing three times for a total of 45 min with TBST (Tris-Buffered Saline Tween-20, TBST) at room temperature, membranes were incubated with secondary antibody goat antimouse $\lg \mathrm{G}(1: 5,000$, Beijing 4A Biotech Co.Ltd) and goat anti-mouse IgG1 (1:10,000, proteintech) for 2h. After washing three times for a total of $45 \mathrm{~min}$ with TBST at room temperature, protein bands were visualized using ECL Blotting Detection Reagents (KPL, 54-61-00).

\section{Statistical analysis}

Each experiment was performed in triplicate, and repeated at least three times. The data are expressed as the mean \pm SD. Statistical analysis was performed using the SPSS 23.0 for Window. Differences were compared between 3-group using one-way ANOVA (analysis of Variance, ANOVA) and 2-group using t tests. The $P \leq 0.05$ was considered to be statistically significant.

\section{Results}

\section{Prediction of microRNA-155 target gene and its signal pathway}

In this study, we extracted 556 target genes from Targetscan database and 700 target genes from mirDB, and 280 genes that are both contained in the two databases were obtained by Venn Mapping (Figure 1. A). The 280 genes were ranked by target score of mirDB, and 49 genes had a target score of 90 or more. GO analysis (Figure 1. B, C, E) and KEGG analysis (Figure 1. F) for the 49 genes in Enrichr database revealed that they were mainly related to autophagy and apoptosis. According to recent studies we chose Rictor and Fos as potential research objects because Rictor was related to autophagy signal pathway and Fos was related to apoptosis signal pathway. We established protein-protein interaction (PPI) network for Rictor (Figure 1. D) and Fos (Figure 1. G). Moreover, the two genes were imported into the TIMER database for more information. Results showed that Rictor and Fos had significant expression differences in a variety of tumor (Figure 1. H, I). Based on the above results, we finally decided to take Rictor and Fos as research objects.

\section{The expression of microRNA-155, Rictor, Fos, Beclin1, LC3, caspase3 and caspase9 via qRT-PCR reaction in gastric cancer and adjacent tissues}

To explore the possible role of microRNA-155 in gastric cancer, we compared the expression of microRNA155 in 43 gastric cancer tissues with that of the adjacent tissues by performing qRT-PCR. When defining $\mathrm{T} / \mathrm{N}>2$ as high expression and $\mathrm{T} / \mathrm{N}<0.5$ as low expression, it revealed that 32 cases with high expression and 11 cases with low expression (Figure 2. A; Table 1.). Then we divided 43 patients into microRNA-155 high expression group (group H, 32 cases) and microRNA-155 low expression group (group L, 11 
cases). Then we detected the expression of Rictor, Fos, Beclin1, LC3, caspase3 and caspase9 by qRT-PCR. We found that Rictor was highly expressed in 12 patients, including 2 in group $\mathrm{H}$ and 10 in group L; the other cases with low expression including 30 in group $\mathrm{H}$ and 1 in group $\mathrm{L}$ (Figure 2. B). There were 33 cases with high expression of Beclin 1 and LC3, including 32 cases in group $\mathrm{H}$ and 1 case in group L; there were 10 cases with low expression were all in group $L$ (Figure 2. C). Fos was normally expressed in 13 patients, including 2 in group $\mathrm{H}$ and 11 in group L; there were 30 patients with low expression or missing expression of Fos, all of them were in group H (Figure 2. D). Caspase3 and caspase9 were highly expressed in 10 cases, all in group $L$; there were 33 cases with low expression, including 32 cases in group $\mathrm{H}$ and 1 case in group $\mathrm{L}$ (Figure 2. E).

\section{Immunohistochemical staining}

To verify the roles of microRNA-155 in clinical samples, immunohistochemistry were used to detect the expression levels of target genes. We found that the expression levels of Rictor (Figure 3. A1, A2), Fos (Figure 3. D1, D2), caspase3 (Figure 3. E1, E2) and caspase9 (Figure 3. F1, F2) were decreased in tumor tissues in group $\mathrm{H}$, whereas the expression of Beclin1 (Figure 3. B1, B2) and LC3 (Figure 3. C1, C2) were significantly upregulated compared with that in adjacent tissues. However in group $L$, we found that the expression levels of Rictor (Figure 3. A3, A4), Fos (Figure 3. D3, D4), Beclin1 (Figure 3. B3, B4), LC3 (Figure 3. C3, C4), caspase3 (Figure 3. E3, E4) and caspase9 (Figure 3. F3, F4) were approximately consistent in the cancer tissues and the adjacent tissues.

\section{Expression of protein encoded by Rictor, Fos, Beclin1, LC3, caspase3 and caspase9 in gastric cancer and adjacent tissues}

To further verify the expression levels of Rictor, Fos, Beclin1, LC3, caspase3 and caspase9 in gastric cancer and adjacent tissues, the corresponding protein levels were detected by western blot. In group $\mathrm{H}$, the expression level of Rictor $(P \otimes 0.001$, Figure 4. A, B) and Fos $(P \otimes 0.001$, Figure 4. E, F) in cancer tissues were significantly lower than those in adjacent tissues; while in group $L$, the level of protein encoded by the two genes was basically the same in cancer tissues and adjacent tissues (Figure 4. A, B, E, F). The expression levels of proteins encoded by Beclin1 $(P \otimes 0.001)$ and LC3 $(P \otimes 0.0005)$ in cancer tissues in group H were significantly higher than those in adjacent tissues (Figure 4. A, C, D), however, the level of protein encoded by the two genes was basically the same in cancer tissues and adjacent tissues in group L (Figure 4. A, C, D). In group $\mathrm{H}$, the expression level of proteins encoded by caspase3 $(P \otimes 0.001)$ and caspase9 $(P \Downarrow 0.0002)$ were lower in cancer tissues than those in the adjacent tissues (Figure 4. E, G, H), but they were basically the same in group L (Figure 4. E, G, H).

\section{Clinical significance of difference in expression levels}

To further explore the clinical significance of the differences in expression levels, all clinicopathologic factors were analyzed in relation to these genes (Table 1; Table 2).

\section{T stage}


According to the expression levels of Rictor, Beclin1/LC3, Fos, caspase3/caspase9, the distribution of all patients in different T stages was analyzed. We found that expression of microRNA-155 ( $P \square 0.017)$ and level of autophagy $(P \otimes 0.001)$ were positive correlated to tumor infiltrate degree, however, the expression of Rictor and Fos and level of apoptosis were negative correlated to tumor infiltrate degree $(P \otimes 0.001)$.

\section{N stage}

According to the expression levels of Rictor, Beclin1/LC3, Fos, caspase3/caspase9, the distribution of all patients in different $\mathrm{N}$ stages was analyzed. We can conclude that with the increase of microRNA-155 ( $P$ 0.05), Beclin1/LC3 expression ( $P \otimes 0.002)$ and the decrease of caspase3/caspase9 expression $(P \otimes 0.002)$, lymph node metastasis increased.

\section{Clinical stage}

According to the expression levels of Rictor, Beclin1/LC3, Fos, caspase3/caspase9, the distribution of all patients in different clinical stages was analyzed. The results showed that in advanced gastric cancer, microRNA-155 was highly expressed ( $P \bigotimes 0.001)$, autophagy was increased $(P \otimes 0.001)$ and apoptosis was reduced $(P \otimes 0.001)$.

\section{Nerve/vascular invasion}

According to the expression levels of Rictor, Beclin1/LC3, Fos, caspase3/caspase9, we analyzed whether the patients had nerve/vascular invension. In conclusion, the patients with vascular invasion had high expression of microRNA-155 ( $P \bigotimes 0.001)$, increased autophagy $(P \otimes 0.001)$ and decreased apoptosis $(P \square$ $0.001)$.

\section{Lauren typing}

According to the expression levels of Rictor, Beclin1/LC3, Fos, caspase3/caspase9, the distribution of all patients in different Lauren type was analyzed. Results informed that expression of microRNA-155, Rictor, Beclin1, LC3, Fos, caspase3 and caspase9 had no clear correlation with Lauren typing $(P \otimes 0.05)$.

\section{Borrmann typing}

According to the expression levels of Rictor, Beclin1/LC3, Fos, caspase3/caspase9, the distribution of all patients in different Borrmann type was analyzed. Cases who had high expression of microRNA-155 ( $P \square$ $0.002)$, Rictor $(P \otimes 0.001)$, low expression of Fos $(P \otimes 0.001)$, increased autophagy and decreased apoptosis were mainly of Borrmann III or IV type.

\section{Discussion}

Previous studies have found that microRNA-155 is abnormally expressed in various tumors and plays a centrol role in the process of tumor initiation, progression, invasion, and metastasis ${ }^{[29-35]}$. Our results indicated that expression of microRNA-155 can be up-regulated in some patients of gastric cancer and 
down-regulated in others. MicroRNA-155 is mainly expressed in B cells, T cells, macrophages and dendritic cells, paticipating in immunity, inflammatory and tumor processes ${ }^{[36]}$. We believe that the abnormal distribution of the above cells in tumor tissues may be one of the reasons for the abnormal expression of microRNA-155. Moreover, Qu ${ }^{[23]}$ reported that microRNA-155 is highly expressed in gastric cancer tissues; Conversely, other groups reported that microRNA-155 was poorly expressed in gastric cancer tissues ${ }^{[24-}$ 26]. To further illustrate the real role of microRNA-155 in gastric cancer, more attention should be paid on this research area.

Our results showed that there was a clear correlation between high expression of microRNA-155 and a variety of clinical characteristics. Clinical characteristics of patients with high expression of microRNA-155 are as follows: T3-4/N2-3 stage, advanced stage, nerve/vascular invasion, poor differentiation, mostly mixed or diffuse type and mostly Borrmann-type III/IV. Based on above characteristics, we inferred that the difference in the expression level of microRNA-155 in cancer tissues was related to different sampling sites, pathological types and clinical stages ${ }^{[24,37,38]}$. Oxidative stress occurring under hypoxic microenvironment has been found to activate autophagy to scavenge reactive oxygen species for protecting cells from damage through upgrading the expression of microRNA-155 ${ }^{[28]}$. Abnormal blood supply in tumor tissues can cause oxidative stress. Generally, the blood supply and nutritional status were different in different parts of the larger lesions, even with local ischemic necrosis. Different pathological types of gastric cancer have different cell proliferation rate and angiogenesis ability. The lower the degree of differentiation, the faster the proliferation rate. When the angiogenesis rate lags behind the tumor growth, it will seriously affect the blood supply and nutrition of the lesion ${ }^{[39-41]}$; The number and distribution of vasa vasorum also varies in different clinical stages. The cellular heterogeneity of tumors is high when tumor has low differentiation, and mitochondrial development tends to be poor, which results in defective aerobic respiration, and causes cancer cells to be in hypoxia state ${ }^{[42-44]}$.

Our results showed that the abnormal expression of microRNA-155 affected autophagy in gastric cancer cells. MTOR is an important factor in the PI3K-Akt-mTOR pathway, which mainly contains two complexes, namely mTORC1 and mTORC2 ${ }^{[45]}$. Rictor is a key component of mTORC2 complex, which plays an important impact on the treatment of tumors ${ }^{[46-48]}$. MTORC2 can activate mTORC1 by phosphorylating Akt and further inhibit autophagy through the PI3K-Akt-mTOR signal pathway. Beclin1 and LC3 are important participants in autophagy. Our findings indicated that microRNA-155 have a lower expression and Rictor have a higher expression in gastric cancer cells in early stages through activating of the PI3K-Akt-mTOR pathway to inhibit autophagy. As gastric cancer progresses, hypoxia and starvation of cancer cells can induce the up-regulation of microRNA-155 expression, inhibit Rictor expression and block the inhibition of autophagy ${ }^{[28]}$, expression of Beclin1 and LC3 increased. The elimination of abnormally expressed proteins, aging or abnormal organelles in cells protects cells from apoptosis or necrosis, and further promotes the progression of gastric cancer.

We also found that abnormal expression of microRNA-155 in gastric cancer affected cell apoptosis. Fos is a member of the Fos protein family, which can be induced by hormones, growth factors, cytokines, heat shock, and oxidative stress. Fos and Jun proteins comprise a heterodimer namely AP-1 (activator protein 1, 
AP-1), an important transcription factors involved in apoptotic pathway and TNF-related signaling pathway ${ }^{[49,50]}$. Thereby AP-1 plays an important role in pathological processes such as proliferation, differentiation, apoptosis, and inflammation of cells ${ }^{[51,52]}$. The level of Fos expression is affected by several factors. Former studies indicated that the expression of Fos can be upregulated by $\mathrm{H}$. pylori infection $[53,54]$ and inhibited by microRNA-7 ${ }^{[55]}$. Our results suggested that expression of Fos, caspase 3 and caspase 9 were negatively regulated by microRNA-155 in gastric cancer. The down-regulation of expression may promote cancer progression, lymph node metastasis and vascular invasion. Low expression of microRNA-155 was observed in early gastric cancer, and there was no significant difference in Fos expression levels between gastric cancer tissues and adjacent tissues. Fos and Jun form AP-1 to promote the progression of gastric cancer by promoting the proliferation and migration of cancer cells ${ }^{[56]}$. In advanced gastric cancer, the expression of microRNA-155 was increased due to ischemia, hypoxia and starvation, then the expression of Fos was decreased or even absent due to the post-transcriptional regulation of microRNA-155, expression of caspase 3 and caspase9 were decreased which play important roles in the apoptosis signal pathway. Due to the apoptosis signal pathway was inhibited, the progression and metastasis of gastric cancer were boosted.

We inferred from our results that abnormal expression of microRNA-155 in gastric tissue may indicate a possible presence of gastric cancer, and its expression level is negatively correlated with clinical stage. Overexpression of microRNA-155 may promote autophagy by inhibiting Rictor expression, meanwhile inhibit apoptosis by inhibiting Fos expression. According to the experimental results, it can be inferred that the overexpression of microRNA-155 may promote the development of gastric cancer in the above ways.

However, this study had a small clinical sample size and may have been biased during case inclusion, and the conclusions have not been validated by in vitro cell experiments.

\section{Conclusion}

In conclusion, the abnormal expression of microRNA-155 may be related to gastric cancer and its expression level is negatively correlated with clinical stage of cancer. The down-regulated expression of Rictor and Fos, enhancement of autophagy and weakening of apoptosis may be related to the overexpression of microRNA-155. MicroRNA-155 may promote the progression of gastric cancer by promoting autophagy and inhibiting apoptosis.

\section{Abbreviations}

qRT-PCR: quantitative real time polymerase chain reaction

TBST: Tris-Buffered Saline Tween-20

ANOVA: analysis of Variance

GO: Gene ontology analysis 
KEGG: Kyote Encyclopedia of Gene and Genome

PPI: protein-protein interaction

AP-1: activator protein 1

\section{Declarations}

\section{Ethics approval and consent to partcipate}

Approval for the research study was obtained from the Lanzhou University Second

Hosptial Ethics Board (project approval number 2017A-044).

\section{Consent for publication}

Not applicable.

\section{Availability of data and materials}

The datasets used and/or analysed during the current study are available from the corresponding author on reasonable request.

\section{Competing interests}

The authors declare that they have no competing interests.

\section{Funding}

Program for International Science and Technology Cooperation Projects of Gansu Province, No. 18YF1WA113; Scientific research project of health industry of Gansu province, No. GSWSKY-2019-32. International S $\varangle$ T Cooperation Program of China, No. 2015DFA31650; National Natural Science Foundation of China, No. 31770537; National Natural Science Foundation of China, 81960673; National Natural Science Foundation of China, No. 81870329.

\section{Authors' Contributions}

GC prepared reagents, collected specimens, conducted qRT-PCR and western blot experiment, analyzed the data, wrote the manuscript; YL performed bioinformatics analysis conducted qRT-PCR; ZJR and YMG collected specimens, conducted qRT-PCR; JM and JMZ co-ordinated and provided the collection of all the human material; LW collected specimens, conducted western blot experiment, analyzed the data. YML and FTT were responsible for the whole experiment design and technical guidance. All authors read and approved the final manuscript.

\section{Authors' information}

Gang Chen chengang201901@163.com 
YuLi 1125367452@qq.com

Zhijian Ren renzhijian126@163.com

Yanmei Gu1367909519@qq.com

Futian Tang tangft@163.com

Jie Mao ery_maoj@Izu.deu.cn

Junmin Zhu zhujm09@126.com

Ling Wang 474750303@qq.com

Yumin Li liym@lzu.edu.cn

\section{Acknowledgements}

We thank everyone of the General Surgery Department and Key Laboratory of Digestive System Tumors of Gansu Province for their sincere help and technical supports.

\section{References}

1 Ferro E, Enrico Bena C, Grigolon S, Bosia C. From Endogenous to Synthetic microRNA Mediated Regulatory Circuits: An Overview. Cells. 2019; doi:10.3390/cells8121540.

2 Wang Y, Hussein AM, Somasundaram L, Sankar R, Detraux D, Mathieu J, et al. microRNAs Regulating Human and Mouse Naïve Pluripotency. Int J Mol Sci. 2019; doi:10.3390/ijms20235864.

3 Stavast CJ, Erkeland SJ. The Non-Canonical Aspects of MicroRNAs: Many Roads to Gene Regulation. Cells. 2019; doi:10.3390/cells8111465.

4 Song J, Zhou S. Post-transcriptional regulation of insect metamorphosis and oogenesis. Cell Mol Life Sci. 2019; doi:10.1007/s00018-019-03361-5.

\section{Karamitopoulou E, Haemmig S, Baumgartner U, Schlup C, Wartenberg M, Vassella E.}

MicroRNA dysregulation in the tumor microenvironment influences the phenotype of pancreatic cancer. Mod Pathol. 2017;30:1116-1125.

6 Liu WL, Wang HX, Shi CX, Shi FY, Zhao LY, Zhao W, et al. MicroRNA-1269 promotes cell proliferation via the AKT signaling pathway by targeting RASSF9 in human gastric cancer. Cancer Cell Int. 2019;19:308.

7 Xiaoyan W, Pais EM, Lan L, Jingrui C, Lin M, Fordjour PA, et al. MicroRNA-155: a Novel Armamentarium Against Inflammatory Diseases. Inflammation. 2017;40:708-716.

8 Peng L, Chen Z, Chen Y, Wang X, Tang N. MIR155HG is a prognostic biomarker and associated with immune infiltration and immune checkpoint molecules expression in multiple cancers. Cancer 
Med. 2019;8:7161-7173.

9 Cui W, Meng W, Zhao L, Cao H, Chi W, Wang B. TGF- $\beta$-induced long non-coding RNA MIR155HG promotes the progression and EMT of laryngeal squamous cell carcinoma by regulating the miR-155-5p/SOX10 axis. Int J Oncol. 2019;54:2005-2018.

10 Takeshige K, Baba M, Tsuboi S, Noda T, Ohsumi Y. Autophagy in yeast demonstrated with proteinasedeficient mutants and conditions for its induction. J Cell Biol. 1992;119:301-11.

11 Anding AL, Baehrecke EH. Cleaning House: Selective Autophagy of Organelles. Dev Cell. 2017;41:10-22.

12 White E, Mehnert JM, Chan CS. Autophagy, Metabolism, and Cancer. Clin Cancer Res. 2015;21:5037-46.

13 Xue H, Yuan G, Guo X, Liu Q, Zhang J, Gao X, et al. A novel tumor-promoting mechanism of IL6 and the therapeutic efficacy of tocilizumab: Hypoxia-induced IL6 is a potent autophagy initiator in glioblastoma via the p-STAT3-MIR155-3p-CREBRF pathway. Autophagy. 2016;12:1129-52.

14 Chung KW, Chung HY. The Effects of Calorie Restriction on Autophagy: Role on Aging Intervention. Nutrients. 2019; doi:10.3390/nu11122923.

15 Jing X, Yang F, Shao C, Wei K, Xie M, Shen H, et al. Role of hypoxia in cancer therapy by regulating the tumor microenvironment. Mol Cancer. 2019;18:157.

16 Li YJ, Lei YH, Yao N, Wang CR, Hu N, Ye WC, et al. Autophagy and multidrug resistance in cancer. Chin J Cancer. 2017;36:52.

17 Yuan R, Hou Y, Sun W, Yu J, Liu X, Niu Y, et al. Natural products to prevent drug resistance in cancer chemotherapy: a review. Ann N Y Acad Sci. 2017;1401:19-27.

18 Tong H, Li T, Qiu W, Zhu Z. Claudin-1 silencing increases sensitivity of liver cancer HepG2 cells to 5fluorouracil by inhibiting autophagy. Oncol Lett. 2019;18:5709-5716.

19 Spirina LV, Avgustinovich AV, Afanas'ev SG, Cheremisina OV, Volkov MY, Choynzonov EL, et al. Molecular mechanism of resistance to chemotherapy in gastric cancers, the role of autophagy. Curr Drug Targets. 2019; doi: 10.2174/1389450120666191127113854.

20 Guo Y, Feng Y, Cui X, Wang Q, Pan X. Autophagy inhibition induces the repolarisation of tumorassociated macrophages and enhances chemosensitivity of laryngeal cancer cells to cisplatin in mice. Cancer Immunol Immunother. 2019;68:1909-1920.

21 Kerr JF, Wyllie AH, Currie AR. Apoptosis: a basic biological phenomenon with wide-ranging implications in tissue kinetics. Br J Cancer. 1972;26:239-57.

22 Elmore S. Apoptosis: a review of programmed cell death. Toxicol Pathol. 2007;35:495-516. 
23 Qu Y, Zhang H, Sun W, Han Y, Li S, Qu Y, et al. MicroRNA-155 promotes gastric cancer growth and invasion by negatively regulating transforming growth factor- $\beta$ receptor 2 . Cancer Sci. 2018;109:618-628.

24 Ma Z, Ma Y, Xia Q, Li Y, Li R, Chang W, et al. MicroRNA-155 expression inversely correlates with pathologic stage of gastric cancerand it inhibits gastric cancer cell growth by targeting cyclin D1. J Cancer Res Clin Oncol. 2016;142:1201-12.

25 Sun S, Sun P, Wang C, Sun T, et al. Downregulation of microRNA-155 accelerates cell growth and invasion by targeting c-myc in human gastric carcinoma cells. Oncol Rep. 2014;32:951-6.

$26 \mathrm{Li} \mathrm{CL}$, Nie H, Wang M, Su LP, Li JF, Yu YY, et al. microRNA-155 is downregulated in gastric cancer cells and involved in cell metastasis. Oncol Rep. 2012;27:1960-6.

27 Yin S, Yang S, Pan X, Ma A, Ma J, Pei H, et al. MicroRNA-155 promotes ox-LDL-induced autophagy in human umbilical vein endothelial cells by targeting the PI3K/Akt/mTOR pathway. Mol Med Rep. 2018;18:2798-2806.

28 Wan G, Xie W, Liu Z, Xu W, Lao Y, Huang N, et al. Hypoxia-induced MIR155 is a potent autophagy inducer by targeting multiple players in the MTOR pathway. Autophagy. 2014;10:70-9.

29 Wang Y, Yan L, Zhang L, Xu H, Chen T, Li Y, et al. NT21 MP negatively regulates paclitaxel-resistant cells by targeting miR-155-3p and miR-155-5p via the CXCR4 pathway in breast cancer. Int $\mathbf{J}$ Oncol. 2018;53:1043-1054.

30 Sannigrahi MK, Sharma R, Panda NK, Khullar M. Role of non-coding RNAs in head and neck squamous cell carcinoma: A narrative review. Oral Dis. 2018;24:1417-1427.

31 Patel GK, Khan MA, Bhardwaj A, Srivastava SK, Zubair H, Patton MC, et al. Exosomes confer chemoresistance to pancreatic cancer cells by promoting ROS detoxification and miR-155-mediated suppression of key gemcitabine-metabolising enzyme, DCK. Br J Cancer. 2017;116:609-619.

32 Pang W, Su J, Wang Y, Feng H, Dai X, Yuan Y, et al. Pancreatic cancer-secreted miR-155 implicates in the conversion from normal fibroblasts to cancer-associated fibroblasts. Cancer Sci. 2015;106:1362-9.

33 Wan YC, Li T, Han YD, Zhang HY, Lin H, Zhang B. MicroRNA-155 enhances the activation of Wnt/ $\beta$ catenin signaling in colorectal carcinoma by suppressing HMG-box transcription factor 1 . Mol Med Rep. 2016;13:2221-8.

34 Mardani R, Jafari Najaf Abadi MH, Motieian M, Taghizadeh-Boroujeni S, Bayat A, Farsinezhad A, et al. MicroRNA in leukemia: Tumor suppressors and oncogenes with prognostic potential. J Cell Physiol. 2019;234:8465-8486.

35 Chen N, Feng L, Qu H, Lu K, Li P, Lv X, et al. Overexpression of IL-9 induced by STAT3 phosphorylation is mediated by miR-155 and miR-21 in chronic lymphocytic leukemia. Oncol Rep. 2018;39:3064-3072. 
36 Elton TS, Selemon H, Elton SM, Parinandi NL. Regulation of the MIR155 host gene in physiological and pathological processes. Gene. 2013;532:1-12.

37 Wang J, Yang K, Zhou L, Minhaowu, Wu Y, Zhu M, et al. MicroRNA-155 promotes autophagy to eliminate intracellular mycobacteria by targeting Rheb. PLoS Pathog. 2013;9:e1003697.

38 Guan C, Yang F, He X, Li T, Yang Q, He H, et al. Clinical significance of microRNA-155 expression in hepatocellular carcinoma. Oncol Lett. 2016;11:1574-1580.

39 Tang E, Wang Y, Liu T, Yan B. Gastrin promotes angiogenesis by activating HIF-1 a/ $\beta$-catenin/VEGF signaling in gastric cancer. Gene. 2019;704:42-48.

40 Lei Z, Chai N, Tian M, Zhang Y, Wang G, Liu J, et al. Novel peptide GX1 inhibits angiogenesis by specifically binding to transglutaminase-2 in the tumorous endothelial cells of gastric cancer. Cell Death Dis. 2018;9:579.

41 Kim SY, Ko YS, Park J, Choi Y, Park JW, Kim Y, et al. Forkhead Transcription Factor FOX01 Inhibits Angiogenesis in Gastric Cancer in Relation to SIRT1. Cancer Res Treat. 2016;48:345-54.

42 Zhao X, Cai A, Peng Z, Liang W, Xi H, Li P, et al. JS-K induces reactive oxygen species-dependent anticancer effects by targeting mitochondriarespiratory chain complexes in gastric cancer. J Cell Mol Med. 2019;23:2489-2504.

43 Proietti S, Cucina A, Minini M, Bizzarri M. Melatonin, mitochondria, and the cancer cell. Cell Mol Life Sci. 2017;74:4015-4025.

44 Skoda J, Borankova K, Jansson PJ, Huang ML, Veselska R, Richardson DR. Pharmacological targeting of mitochondria in cancer stem cells: An ancient organelle at the crossroad of novel anti-cancer therapies. Pharmacol Res. 2019;139:298-313.

45 Hua H, Kong Q, Zhang H, Wang J, Luo T, Jiang Y. Targeting mTOR for cancer therapy. J Hematol Oncol. 2019;12:71.

46 Kim ST, Kim SY, Klempner SJ, Yoon J, Kim N, Ahn S, et al. Rapamycin-insensitive companion of mTOR (RICTOR) amplification defines a subset of advanced gastric cancer and is sensitive to AZD2014-mediated mTORC1/2 inhibition. Ann Oncol. 2017;28:547-554.

47 Akgül S, Li Y, Zheng S, Kool M, Treisman DM, Li C, et al. Opposing Tumor-Promoting and-Suppressive Functions of Rictor/mTORC2 Signaling in Adult Glioma and Pediatric SHH Medulloblastoma. Cell Rep. 2018;24:463-478.

48 Holmes B, Benavides-Serrato A, Freeman RS, Landon KA, Bashir T, Nishimura RN, et al. mTORC2/AKT/HSF1/HuR constitute a feed-forward loop regulating Rictor expression and tumor growth in glioblastoma. Oncogene. 2018;37:732-743. 
49 Yang W, Zhao S, Wu B, Xu J, Wu Z, Guo J, et al. BATF2 inhibits chemotherapy resistance by suppressing AP-1 in vincristine-resistant gastric cancer cells. Cancer Chemother Pharmacol. 2019;84:12791288.

50 Zhang LZ, Man QW, Liu JY, Zhong WQ, Zheng YY, Zhao YF, et al. Overexpression of Fra-1, c-Jun and cFos in odontogenic keratocysts: potential correlation with proliferative and anti-apoptotic activity. Histopathology. 2018;73:933-942.

51 Tyagi A, Vishnoi K, Kaur H, Srivastava Y, Roy BG, Das BC, et al. Cervical cancer stem cells manifest radioresistance: Association with upregulated AP-1 activity. Sci Rep. 2017;7:4781.

52 Yadav S, Kalra N, Ganju L, Singh M. Activator protein-1 (AP-1): a bridge between life and death in lung epithelial (A549) cells under hypoxia. Mol Cell Biochem. 2017;436:1-12.

53 Wu D, Cao M, Peng J, Li N, Yi S, Song L, et al. The effect of trimethylamine N-oxide on Helicobacter pyloriinduced changes of immunoinflammatory genes expression in gastric epithelial cells. Int Immunopharmacol. 2017;43:172-178.

54 Asim M, Chaturvedi R, Hoge S, Lewis ND, Singh K, Barry DP, et al. Helicobacter pylori induces ERKdependent formation of a phospho-c-Fos c-Jun activator protein-1 complex that causes apoptosis in macrophages. J Biol Chem. 2010;285:20343-20357.

55 Zhao XD, Lu YY, Guo H, Xie HH, He LJ, Shen GF, et al. MicroRNA-7/NF-kB signaling regulatory feedback circuit regulates gastric carcinogenesis. J Cell Biol. 2015;210:613-27.

56 Fu J, Cheng L, Wang Y, Yuan P, Xu X, Ding L, et al. The RNA-binding protein RBPMS1 represses AP-1 signaling and regulates breast cancer cell proliferation and migration. Biochim Biophys Acta. 2015;1853:113.

\section{Tables}




\begin{tabular}{|c|c|c|c|c|}
\hline & & & Sig & ficance \\
\hline & $\mathrm{n}(\%)$ & $\mathrm{n}(\%)$ & $\mathrm{X}^{2}$ & P-value \\
\hline Age & & & 0.430 & 0.512 \\
\hline 059years & $11(34.3)$ & $5(45.5)$ & & \\
\hline$\geq 59$ years & $21(65.7)$ & $6(54.5)$ & & \\
\hline Gender & & & 0.425 & 0.514 \\
\hline Male & $23(71.9)$ & $9(81.8)$ & & \\
\hline Female & $9(28.1)$ & $2(18.20$ & & \\
\hline Degree of tumor cell differentiation & & & 1.901 & 0.168 \\
\hline Moderate-to-well differentiated & $10(31.3)$ & $6(54.5)$ & & \\
\hline Poorly differentiated & $22(68.7)$ & $5(45.5)$ & & \\
\hline TNM stage & & & 5.731 & $0.017^{\mathrm{a}}$ \\
\hline $\mathrm{I}+\mathrm{II}$ & $16(50.0)$ & $10(90.9)$ & & \\
\hline $\mathrm{III}+\mathrm{IV}$ & $16(50.0)$ & $1(9.1)$ & & \\
\hline Clinical stage & & & 33.136 & $0.000^{b}$ \\
\hline Early & $1(3.1)$ & $10(90.9)$ & & \\
\hline Progressive & $31(96.9)$ & $1(9.1)$ & & \\
\hline Lymph node metastasis & & & 11.711 & $0.001^{\mathrm{C}}$ \\
\hline Negative & $10(31.3)$ & $10(90.9)$ & & \\
\hline Positive & $22(68.7)$ & $1(9.1)$ & & \\
\hline Venous invasion & & & 12.589 & $0.000^{\mathrm{d}}$ \\
\hline Negative & $7(21.9)$ & $9(81.8)$ & & \\
\hline Positive & $25(78.1)$ & $2(18.2)$ & & \\
\hline Lauren type & & & 3.220 & 0.200 \\
\hline Intestinal & $13(40.6)$ & $7(63.6)$ & & \\
\hline Diffuse & $12(37.5)$ & $1(9.1)$ & & \\
\hline Mixed & $7(21.9)$ & $3(27.3)$ & & \\
\hline Borrmann type & & & 9.232 & $0.002^{\mathrm{e}}$ \\
\hline $\mathrm{I}+\mathrm{II}$ & $2(6.3)$ & $5(45.5)$ & & \\
\hline $\mathrm{III}+\mathrm{IV}$ & $30(93.7)$ & $6(54.5)$ & & \\
\hline
\end{tabular}

Table 1. MicroRNA-155 and clinicopathologic factors. 


\begin{tabular}{|c|c|c|c|c|c|c|c|c|c|c|c|c|c|c|c|c|}
\hline & \multicolumn{4}{|c|}{ Rictor } & \multicolumn{4}{|c|}{ Beclin1/LC3 } & \multicolumn{4}{|c|}{ Fos } & \multicolumn{4}{|c|}{ Caspase $3 / 9$} \\
\hline & \multicolumn{2}{|c|}{ expression } & \multicolumn{2}{|c|}{ significance } & \multicolumn{2}{|c|}{ expression } & \multicolumn{2}{|c|}{ significance } & \multicolumn{2}{|c|}{ expression } & \multicolumn{2}{|c|}{ significance } & \multicolumn{2}{|c|}{ expression } & \multicolumn{2}{|c|}{ significance } \\
\hline & High & Low & $\mathrm{X}^{2}$ & $P$ & High & Low & $\mathrm{X}^{2}$ & $P$ & High & Low & $\mathrm{X}^{2}$ & $P$ & High & Low & $\mathrm{X}^{2}$ & $P$ \\
\hline $\mathrm{T}$ stage & & & 40.515 & 0 & & & 37.906 & 0 & & & 43 & 0 & & & 31.03 & 0.000 \\
\hline 1 & 11 & & & & 1 & 10 & & & 11 & & & & 9 & 2 & & \\
\hline 2 & 1 & 1 & & & 2 & & & & 2 & & & & 1 & 1 & & \\
\hline 3 & & 20 & & & 20 & & & & & 20 & & & & 20 & & \\
\hline 4 & & 10 & & & 10 & & & & & 10 & & & & 10 & & \\
\hline N stage & & & 13.978 & 0.003 & & & 14.985 & 0.002 & & & 12.157 & 0.007 & & & 14.985 & 0.002 \\
\hline 0 & 11 & 9 & & & 10 & 10 & & & 11 & 9 & & & 10 & 10 & & \\
\hline 1 & 1 & 8 & & & 9 & & & & 2 & 7 & & & & 9 & & \\
\hline 2 & & 6 & & & 6 & & & & & 6 & & & & 6 & & \\
\hline 3 & & 8 & & & 8 & & & & & 8 & & & & 8 & & \\
\hline $\begin{array}{l}\text { Clinical } \\
\text { stage }\end{array}$ & & & 38.185 & 0 & & & 37.906 & 0 & & & 34.111 & 0 & & & 28.403 & 0 \\
\hline Early & 11 & & & & 1 & 10 & & & 11 & & & & 9 & 2 & & \\
\hline Progressive & 1 & 31 & & & 32 & & & & 2 & 30 & & & 1 & 31 & & \\
\hline $\begin{array}{l}\text { Venous } \\
\text { invasion }\end{array}$ & & & 21.128 & 0 & & & 21.989 & 0 & & & 17.923 & 0 & & & 15.543 & 0 \\
\hline Negative & 11 & 5 & & & 6 & 10 & & & 11 & 5 & & & 9 & 7 & & \\
\hline Positive & 1 & 26 & & & 27 & & & & 2 & 25 & & & 1 & 26 & & \\
\hline $\begin{array}{l}\text { Lauren } \\
\text { type }\end{array}$ & & & 9.086 & 0.011 & & & 0.597 & 0.742 & & & 4.488 & 0.106 & & & 5.891 & 0.053 \\
\hline Intestinal & 10 & 10 & & & 15 & 5 & & & 8 & 12 & & & 8 & 12 & & \\
\hline Diffuse & 1 & 12 & & & 10 & 5 & & & 1 & 12 & & & 1 & 12 & & \\
\hline $\begin{array}{l}\text { Mixed } \\
\text { Borrmann }\end{array}$ & 1 & 9 & & & 8 & 2 & & & 4 & 6 & & & 1 & 9 & & \\
\hline type & & & 13.887 & 0 & & & 5.38 & 0.02 & & & 12.202 & 0 & & & 18.276 & 0 \\
\hline $\mathrm{I}+\mathrm{II}$ & 6 & 1 & & & 3 & 4 & & & 6 & 1 & & & 6 & 1 & & \\
\hline $\mathrm{III}+\mathrm{IV}$ & 6 & 30 & & & 30 & 6 & & & 7 & 29 & & & 4 & 32 & & \\
\hline
\end{tabular}

Table 2. Gene expression and clinicopathologic factors.

\section{Figures}




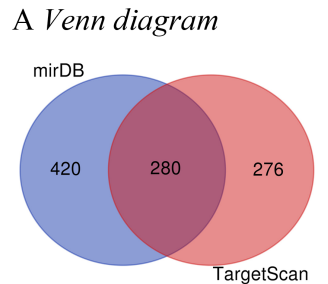

D PPI of Rictor

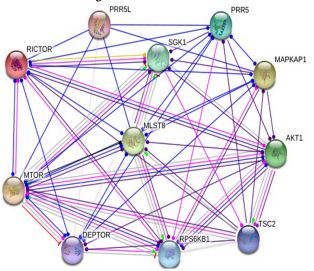

G PPI of Fos

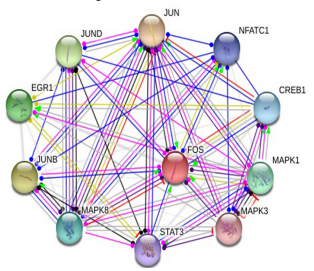

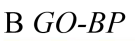

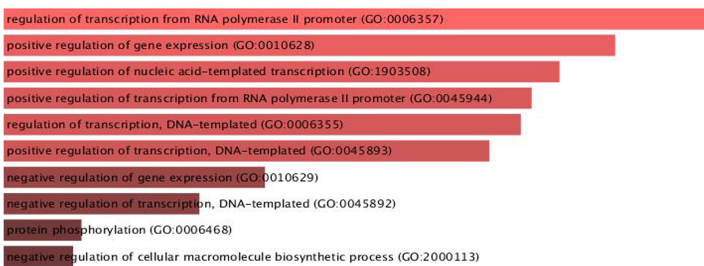

E $\mathrm{GO}-\mathrm{CC}$

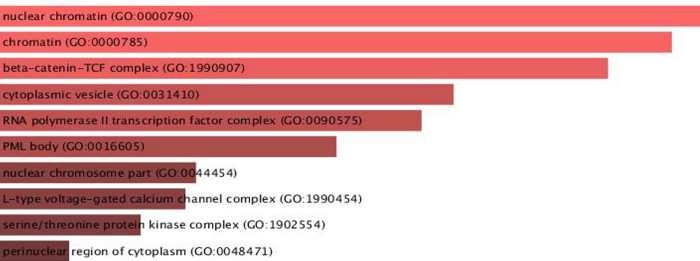

$\mathrm{H}$ Expression of Rictor

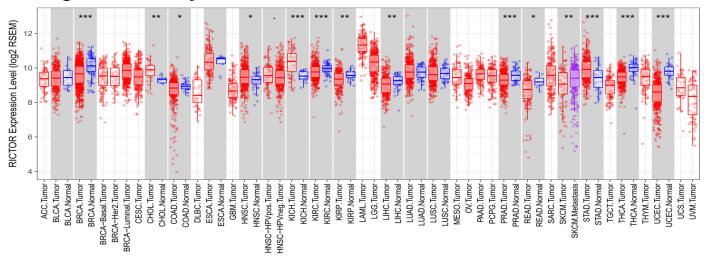

C $G O-M F$

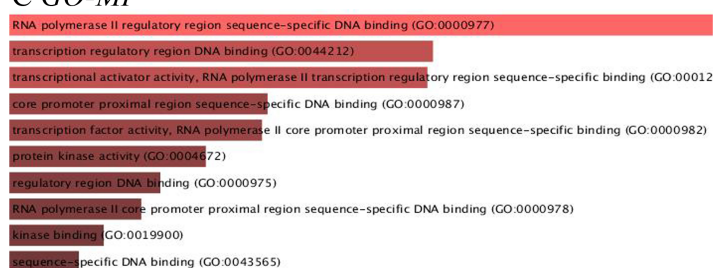

F $K E G G$

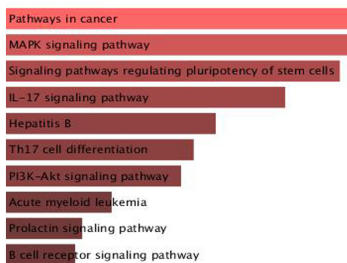

I Expression of Fos

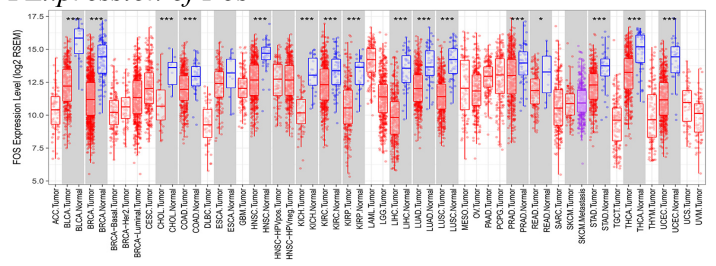

Figure 1

Results of bioinformatics analysis. A. Venn mapping of the prediction results of target genes in mirDB and TargetScan databases; B, C, E, F. GO and KEGG analyzation of the 49 target genes whose mirDB target score $>90$ in Enrichr database; D, G. The protein-protein interaction network of Rictor and Fos in String database; $\mathrm{H}$, I. Analyzation of the expression differences between tumor tissues and paracancer tissues of Rictor and Fos in TIMER database.

\section{A microRNA-155}

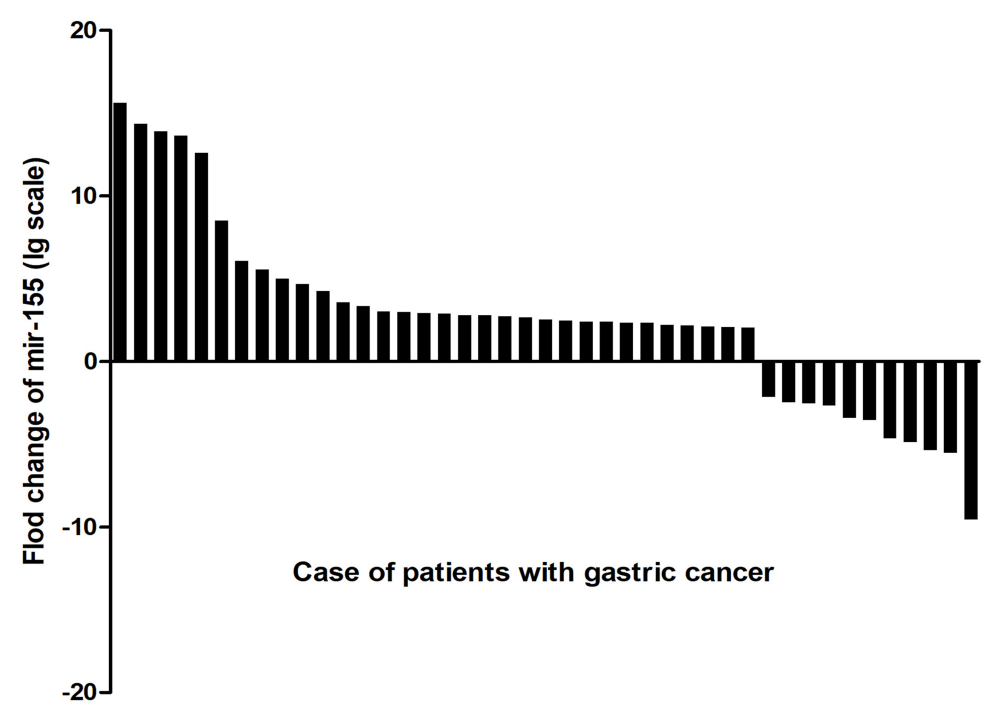

B Rictor

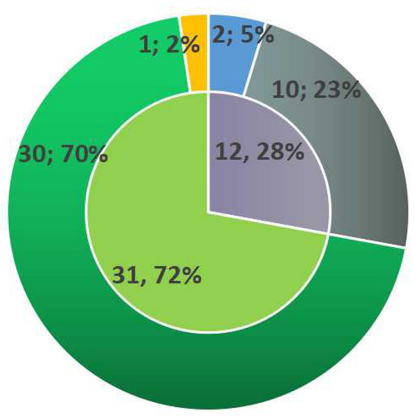

D Fos

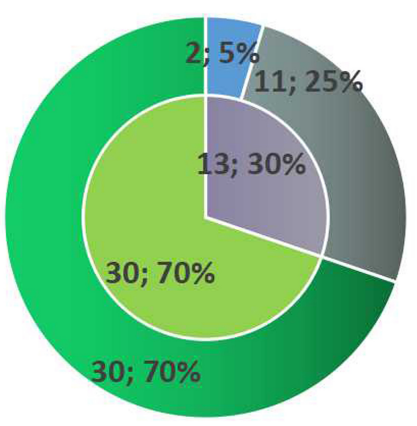

C Beclin1/LC3

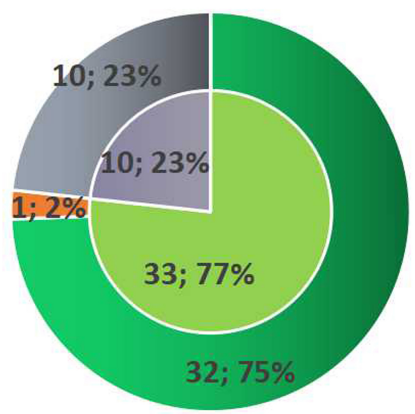

E caspase3/9

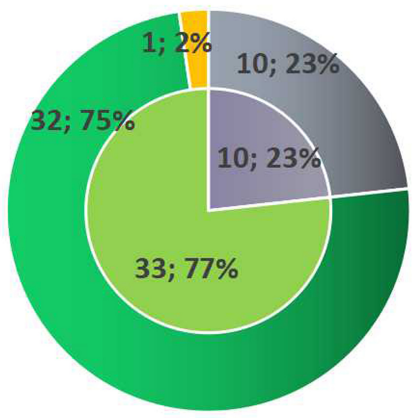


Figure 2

Results of qRT-PCR of microrna-155, Rictor, Beclin1, LC3, Fos, caspase3 and caspase9. A. Expression of microrna-155 in cancerous and paracancer tissues of 43 patients. B-E. Expression of Rictor, Beclin1, LC3, Fos, caspase3, caspase9 and their distribution in group $\mathrm{H}$ or group $\mathrm{L}$.

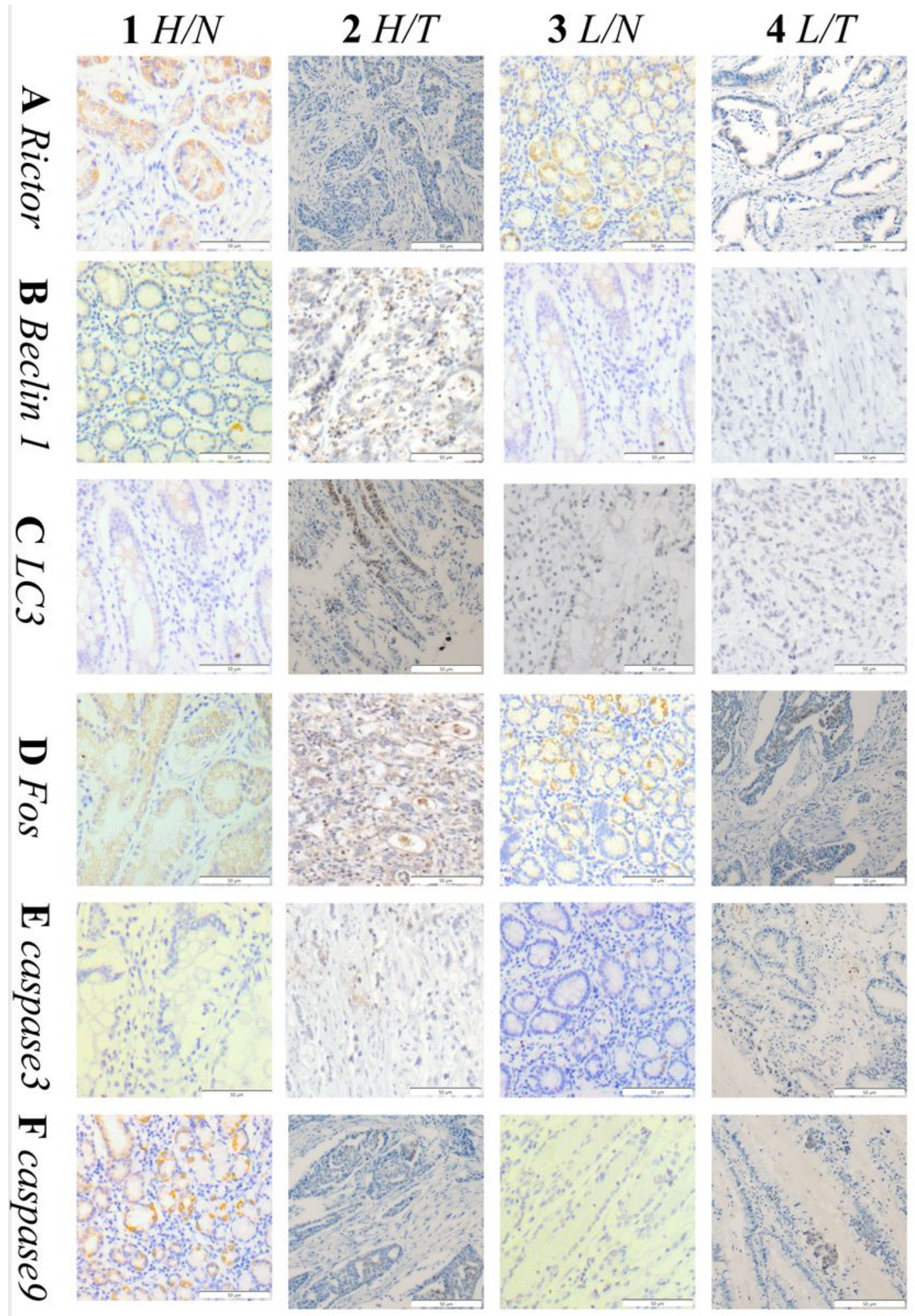

\section{Figure 3}


Immunohistochemical results of Rictor, Beclin1, LC3, Fos, caspase3, caspase9. In group H, Rictor (A1, A2), Fos (D1, D2), caspase3 (E1, E2) and caspase9 (F1, F2) were down-regulated, Beclin1 (B1, B2) and LC3 (C1, C2) were up-regulated. In group L, expression of Rictor (A3, A4), Fos (D3, D4), Beclin1 (B3, B4), LC3 (C3, C4), caspase3 (E3, E4) and caspase9 (F3, F4) were approximately the same in cancer tissues and adjacent tissues.

A

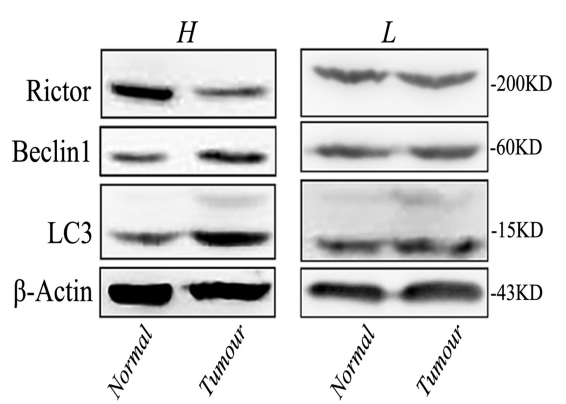

$\mathrm{E}$

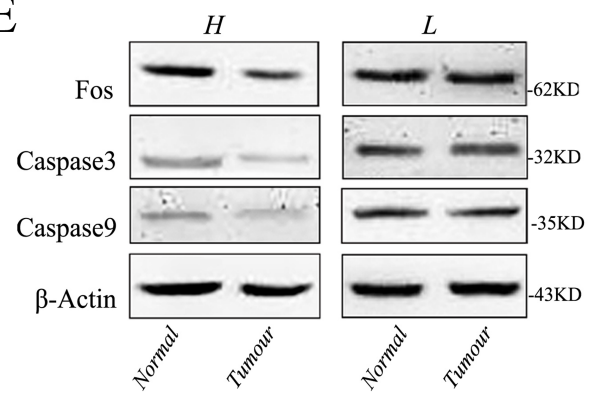

B Rictor

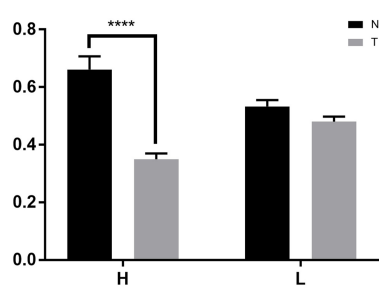

F Fos

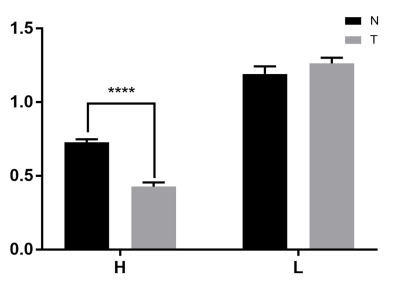

C Beclin1

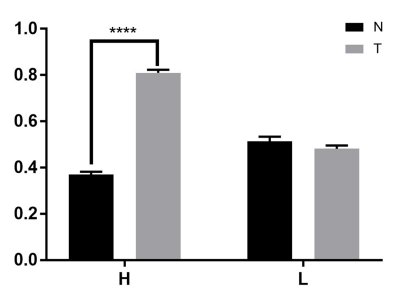

G caspase3

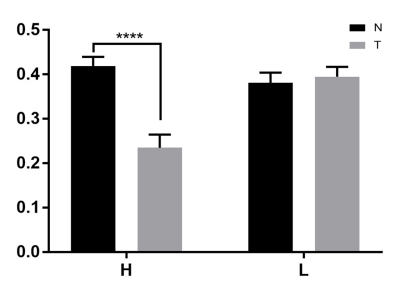

$\mathrm{D} L C 3$

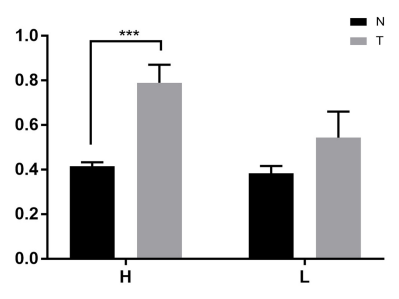

H caspase9

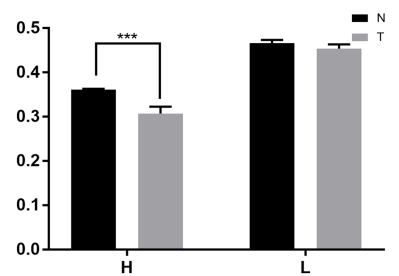

\section{Figure 4}

Results of WB of microrna-155, Rictor, Beclin1, LC3, Fos, caspase3 and caspase9. A. Expression of Rictor, Beclin1 and LC3 in cancer tissues and adjacent tissues; B-D. Differences of expression levels between group $\mathrm{H}$ and group L. E. Expression of Fos, caspase3 and caspase9 in cancer tissues and adjacent tissues; F-H. Differences of expression levels between group $\mathrm{H}$ and group $\mathrm{L}$. 